\title{
MANUAL ADJUSTMENT OF BRAIN EXTRACTION PARAMETERS IN A VOLUMETRIC STUDY
}

\author{
Ivan Dimitrov ${ }^{1}$, Radoslav Georgiev ${ }^{2}$, Nataliya Usheva ${ }^{3}$ \\ ${ }^{1}$ Head of Affiliate Sliven, Medical University of Varna \\ ${ }^{2}$ Department of Imaging Diagnostics and Radiotherapy, Medical University of Varna \\ ${ }^{3}$ Department of Social Medicine and Health Care Organisation, \\ Medical University of Varna
}

\begin{abstract}
INTRODUCTION: Brain volumetry is a contemporary method used in the scientific and clinical research in neurodegenerative diseases. The process can be fully automated but it allows some parameters to be manually adjusted in order to minimize errors. The purpose of the present study is to analyze the use of additional settings in the process of extracting brain tissue from the skull in volumetric assessments performed using FSL-SIENAX, to point out the most frequently used ones, and to provide recommendations for their application.

MATERIAL AND METHODS: 3DT1 MRI scans of 51 patients with multiple sclerosis were processed. After a conversion from the native format, brain tissue was extracted using the BET procedure. Multiple experiments were done using different parameters followed by a visual assessment of the results. Optimal values were chosen for each case. Descriptive statistical analysis was performed.

RESULTS: Manual corrections of the default settings of BET were made in all studied cases. The most frequently applied parameter (100\% of cases) was “-f", which adjusts the aggressiveness of the algorithm, followed by "-B" (51\%), which reduces the bias field and neck voxels, “-R" (31.4\%), multiple iterations of the algorithm, “-g” (25.5\%), correction of the vertical gradient, “-S” (2\%), removal of wrongfully identified optic nerves and eyeballs.

CONCLUSION: The fully automatic volumetric assessment of the brain performed by FSL-SIENAX accelerates the workflow, but may lead to imperfections in the results. Manual adjustment trials may begin with the “-f” parameter, followed by “-B”, “-R”, “-g”, and combinations between them.
\end{abstract}

Keywords: BET, brain extraction, multiple sclerosis, SIENAX, volumetric study

Address for correspondence:

Assoc. Prof. Ivan Dimitrov, $M D, P h D$

St. Marina University Hospital of Varna

Medical University of Varna

1, Hristo Smirnenski Str.

9010 Varna, Bulgaria

e-mail: indimitrov@yahoo.com

Received: August 2, 2015

Accepted: September 15, 2015

\section{INTRODUCTION}

Brain volumetry is a contemporary method used in scientific research and in clinical trials of potential drugs in the field of neurodegenerative disorders, including but not limited to Alzheimer's disease, predementia states, and multiple sclerosis (MS) $(1,2)$. It is performed using specialized software products, after feeding in images from magnetic resonance tomography (MRI), usually 3DT1 scans. Rou- 
tine MRI cannot directly provide numeric data reflecting the volume of the brain, the white and gray matter, or specific subcortical structures. This information is often needed to precisely quantify macroscopic morphologic changes, and that is why it has to be obtained after the images undergo software processing. Several programs have been developed for this purpose, and some of them are free to use. SIENA (Structural Image Evaluation, using Normalization, of Atrophy), part of FSL (FMRIB Software Library) (3), is a procedure which computes the percentage of brain volume change between two MRI assessments of the same person, performed at different moments of time. SIENA uses a series of routines. Especially important among them is the Brain Extraction Tool (BET), which aims at removing all tissues except the brain. In SIENA, voxel parameters of atrophy can be projected on standard space in order to perform statistical comparison between multiple assessed subjects (4). A variant of SIENA called SIENAX has been developed. It is capable of computing brain volume from a single MRI assessment, normalized for the size of the skull (5). SIENAX, as well as SIENA, extracts the brain from the skull using BET. The workflow can be completely automated, but it allows manual tuning of some parameters, including those of BET, so as to minimize possible errors $(6,7)$.

It is known that the automated methods which isolate brain tissues in MRI are prone to be influenced by different factors, such as inhomogeneities of the signal, type ofthe image set, regional anatomy, and age and diagnosis of subjects (8). For this reason, manual control is often recommended and research is being done for the development of novel automated methods which would be precise even when default settings are used. Wang et al. (9) have proposed a method which combines two approaches: atlas-based and deformable-surface-based, that is guided by local intensity information and population-specific prior information learned from a set of real brain images. Having compared their method with other available techniques, they demonstrate its superiority on all testing datasets, as well as its robustness and effectiveness. Lemieux et al. (10) have proposed an automated algorithm segmenting the brain from T1 MRI scans, which uses thresholding and morphological operations, in order to achieve results similar to those of a semi-automated method. Another fully automatic brain extraction method, "brain tissue extraction method using erosiondilation treatment (BREED)", has been proposed by Miura et al. (11). The authors report accuracy of the method of approximately $97 \%$ for either simulated or subject data. Rehm et al. (12) have also introduced their proprietary method (McStrip) for brain/nonbrain segmentation on T1 MRI. Being a more complex hybrid method, it was able to outperform SPM2, BET, and BSE, on head-to-head comparison (13). Another hybrid method combining watershed algorithms and deformable surface models was proposed by Ségonne et al. (14) who claim that their method outperforms other available tools.

While some authors and research teams focus on the development of new methods, others search for the best settings that allow existing techniques to produce better results. Shattuck et al. (15) have compared different brain extraction methods, including BET, using their own dataset and validation method, and have concluded that all algorithms can achieve satisfactory results, but need proper selection of parameters. According to Popescu et al. (16) BET may perform reasonably well on most 3DT1 images of MS patients, but the performance can be improved substantially by adjusting some parameters. The authors encourage the use of options "- $\mathrm{B}$ " and "- $\mathrm{f}=0.1$ " after removal of the neck slices, as they have found this to work best for all acquisition protocols.

The aim of the present study is to analyze the use of additional settings for the BET algorithm in the process of extracting brain tissue from the skull in a volumetric assessment performed using FSLSIENAX, to point out the most frequently used ones, and to provide recommendations for their application.

\section{MATERIAL AND METHODS}

3DT1 MRI scans of 51 patients with multiple sclerosis were processed. Images were obtained using a 1.5 T MRI scanner, Signa HDxt (General Electric, Milwaukee, WI, USA), in St. Marina University Hospital in Varna, Bulgaria. DICOM series were converted to an appropriate format for further processing using MRIConvert (Lewis Center for Neuroimaging, University of Oregon, USA). After conversion from the native format, brain tissue was extracted using 
the BET procedure, FSL running on an Intel ${ }^{\circ}$ Core i7 920 based computer with 32 GB of RAM and CentOS 6 operating system. Multiple experiments were performed using different parameters, followed by visual assessment of the results. Optimal values were identified for each case. Results were summarized using descriptive statistics, providing the percentage of cases where each parameter was used.

\section{RESULTS}

All 51 cases were processed successfully by the software, the routines finishing without an error output. Nevertheless, after visual inspection, manual corrections to the default settings of BET were considered necessary and were applied in all cases. The most frequent (in $100 \%$ of cases) was ,- $\mathrm{f}^{\text {“ }}$, adjusting the aggressiveness of the algorithm, followed by „-B“ (51\%), which reduces errors due to bias field and neck voxels. Third according to the frequency of use was „- $\mathrm{R}^{\text {“ }}(31,4 \%)$, performing several iterations, fourth was "-g“ (25,5\%), which corrects vertical gradient. The „-S“ parameter, aiming at removing erroneously identified optic nerves and eyeballs, was used in $2 \%$ of the cases. On our machine, BET with default settings took several seconds to complete, ,-f“, ,-g“ and ”- $\mathrm{R}$ “ caused no significant slowing, while „-B“ and „-S“ took more than 20 min. per case.

Examples of delineation of the brain using default and manually adjusted parameters of BET are shown on Fig. 1 and 2.

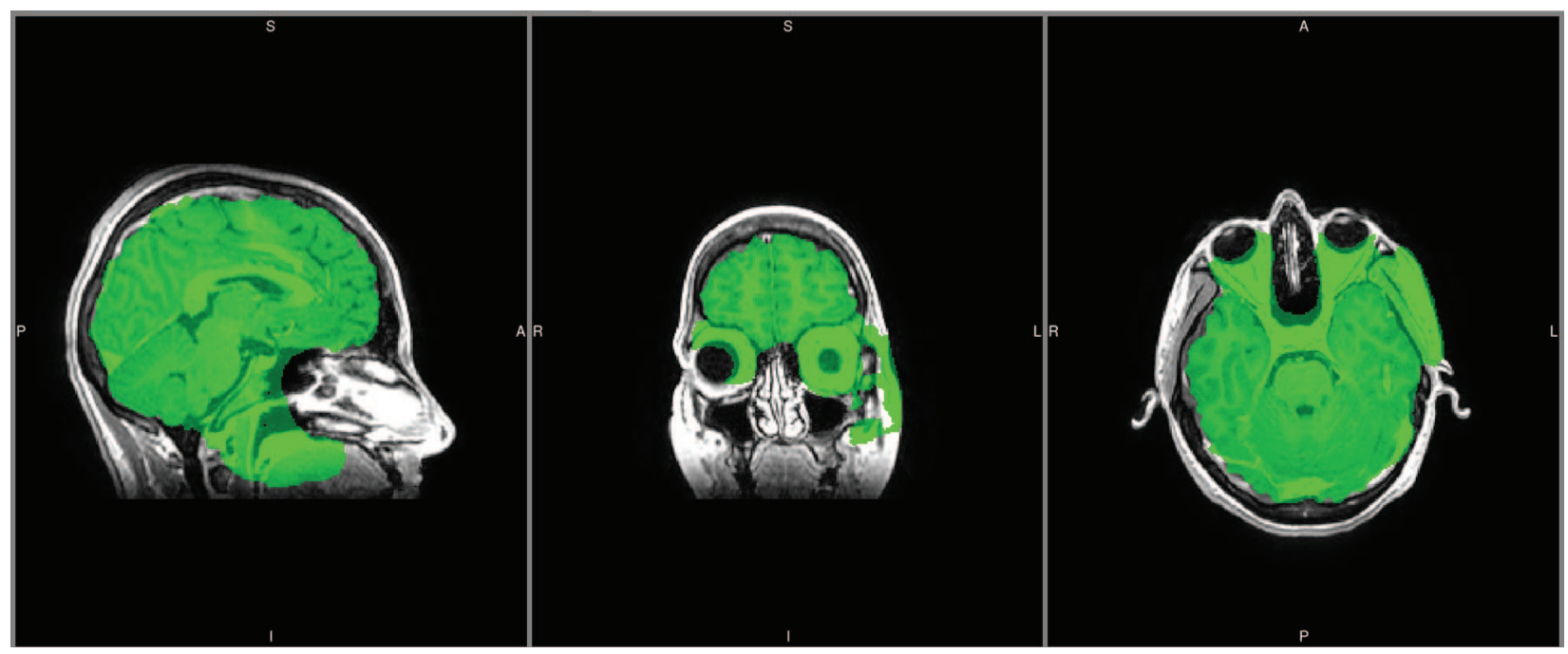

Fig.1. Delineation of brain using default parameters of BET: low precision, errors in tissue identification

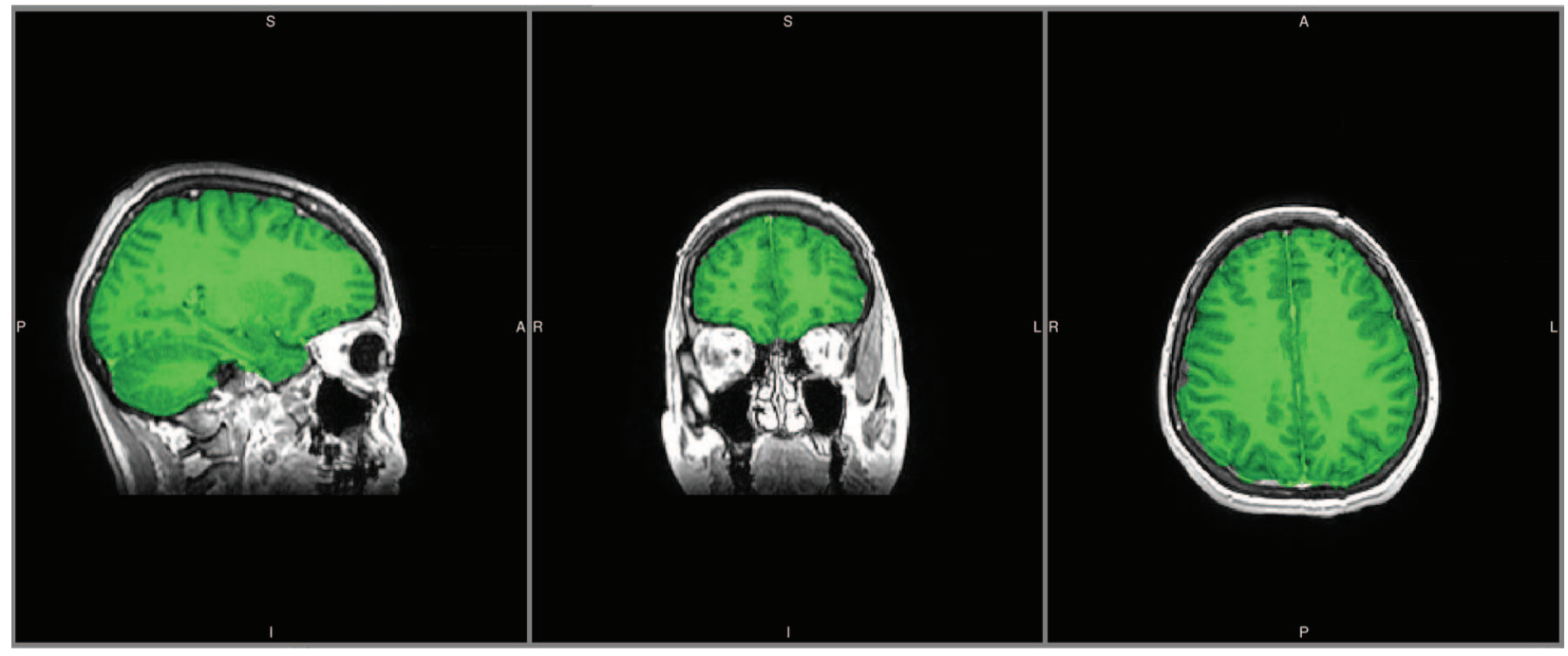

Fig.2. Delineation of brain using manually adjusted parameters of BET (“- $B$ ”): high precision is achieved 
Ivan Dimitrov, Radoslav Georgiev, Nataliya Usheva

\section{DISCUSSION}

In our study we have used a well-established method (BET, part of FSL) for removal of the skull and other tissues which are not needed in the process of calculating the volume of the brain. Such stripping of the brain from other tissues can be done automatically when SIENA (SIENAX) procedure is called with its default values. As such practice may lead to inconsistencies and bias, we checked what the default values would produce, and found problems of different severity in all our cases. Adjustment of parameters was then made and only after numerous experiments with the settings, the results became satisfactory. In our study some parameters showed better potential for successful correction of errors than others. We support the recommendations of Popescu et al. (16) for the use of the „- $\mathrm{B}^{\prime \prime}$ parameter in all cases, as well as for setting the "-f" parameter to a slightly higher aggressiveness than by default. On the other hand, we would like to underline that with some of these parameters applied, image processing may require significant amounts of time. While in our study $\mathrm{BET}$ was completing in several seconds using the default parameters, adding „-B“ or „-S“ prolonged the duration to over $20 \mathrm{~min}$. In some cases it seemed better to experiment with the simple, less time-consuming parameters („-f“, „-g“, etc.) and, if the result was acceptable, to try to avoid the usually more effective but time-consuming „-B“. We found „-B“ to be more effective than „-S“ in removing optic nerves and eyeballs in most of the cases, which explains the relatively low use of the "-S“ parameter in our study.

Regardless of the specific aims of the volumetric analysis in different studies, increasing the accuracy of the method remains a priority (14). In research and in a clinical setting, there is a need for critical reappraisal of the results of the automated analysis (13). Valid normative data and parameters for determining pathologic cut-off values for brain atrophy in MS are subject to current research (17). The need for obtaining correct quantitative data becomes more and more critical, as we are witnessing a stronger interest towards therapies which modify the aggressiveness of pathological processes in the brain, such as that in MS.

\section{CONCLUSION}

The fully automatic volumetric assessment of the brain performed by FSL-SIENAX accelerates the workflow and makes it easier to accomplish, but may lead to imperfections in the results. Our data shows the need for intervention in $100 \%$ of the cases. Correction trials may begin by adjusting the “-f" parameter, followed by “-B", “-R", “-g”, and then some feasible combinations between them can be applied. It is important to underline that the method we applied could be recommended for conducting analysis in similar scenarios. At the same time, some imperfections of the existing volumetric methods bring a strong motivation for continuing the research for novel, more accurate and robust techniques for quantitative assessment of normal and pathologically altered MRI morphology of the brain.

\section{REFERENCES}

1. Giorgio A, De Stefano N. Clinical use of brain volumetry. J Magn Reson Imaging. 2013 Jan;37(1):1-14.

2. Radue EW, Barkhof F, Kappos L, Sprenger T, Haring DA, de Vera A, et al. Correlation between brain volume loss and clinical and MRI outcomes in multiple sclerosis. Neurology. $2015 \mathrm{Feb}$ 24;84(8):784-793.

3. Smith SM, Zhang Y, Jenkinson M, Chen J, Matthews PM, Federico A, et al. Accurate, robust, and automated longitudinal and cross-sectional brain change analysis. Neuroimage. 2002 Sep;17(1):479-489.

4. Smith SM, Jenkinson M, Woolrich MW, Beckmann CF, Behrens TE, Johansen-Berg H, et al. Advances in functional and structural MR image analysis and implementation as FSL. Neuroimage. 2004;23 Suppl 1:S208-219.

5. Smith SM. Fast robust automated brain extraction. Hum Brain Mapp. 2002 Nov;17(3):143-155.

6. Battaglini M, Smith SM, Brogi S, De Stefano N. Enhanced brain extraction improves the accuracy of brain atrophy estimation. Neuroimage. $2008 \mathrm{Apr}$ 1;40(2):583-589.

7. Jasperse B, Valsasina P, Neacsu V, Knol DL, De Stefano N, Enzinger C, et al. Intercenter agreement of brain atrophy measurement in multiple sclerosis patients using manually-edited SIENA and SIENAX. J Magn Reson Imaging. 2007 Oct;26(4):881-885. 
Manual adjustment of brain extraction parameters in a volumetric study

8. Fennema-Notestine C, Ozyurt IB, Clark CP, Morris S, Bischoff-Grethe A, Bondi MW, et al. Quantitative evaluation of automated skull-stripping methods applied to contemporary and legacy images: effects of diagnosis, bias correction, and slice location. Hum Brain Mapp. 2006 Feb;27(2):99-113.

9. Wang Y, Nie J, Yap PT, Li G, Shi F, Geng X, et al. Knowledge-guided robust MRI brain extraction for diverse large-scale neuroimaging studies on humans and non-human primates. PLoS One. 2014;9(1):e77810.

10. Lemieux L, Hagemann G, Krakow K, Woermann FG. Fast, accurate, and reproducible automatic segmentation of the brain in T1-weighted volume MRI data. Magn Reson Med. 1999 Jul;42(1):127-135.

11. Miura N, Taneda A, Shida K, Kawashima R, Kawazoe $\mathrm{Y}$, Fukuda $\mathrm{H}$, et al. Automatic brain tissue extraction method using erosion-dilation treatment (BREED) from three-dimensional magnetic resonance imaging T1-weighted data. J Comput Assist Tomogr. 2002 Nov-Dec;26(6):927-932.

12. Rehm K, Schaper K, Anderson J, Woods R, Stoltzner S, Rottenberg D. Putting our heads together: a consensus approach to brain/non-brain segmentation in T1-weighted MR volumes. Neuroimage. 2004 Jul;22(3):1262-1270.

13. Boesen K, Rehm K, Schaper K, Stoltzner S, Woods $\mathrm{R}$, Luders E, et al. Quantitative comparison of four brain extraction algorithms. Neuroimage. 2004 Jul;22(3):1255-1261.

14. Segonne F, Dale AM, Busa E, Glessner M, Salat D, Hahn HK, et al. A hybrid approach to the skull stripping problem in MRI. Neuroimage. 2004 Jul;22(3):1060-1075.

15. Shattuck DW, Prasad G, Mirza M, Narr KL, Toga AW. Online resource for validation of brain segmentation methods. Neuroimage. 2009 Apr 1;45(2):431-439.

16. Popescu V, Battaglini M, Hoogstrate WS, Verfaillie SC, Sluimer IC, van Schijndel RA, et al. Optimizing parameter choice for FSL-Brain Extraction Tool (BET) on 3D T1 images in multiple sclerosis. Neuroimage. 2012 Jul 16;61(4):1484-1494.

17. De Stefano N, Stromillo ML, Giorgio A, Bartolozzi ML, Battaglini M, Baldini M, et al. Establishing pathological cut-offs of brain atrophy rates in multiple sclerosis. J Neurol Neurosurg Psychiatry. 2015;0:1-7. 